\title{
ERRATUM
}

\section{Erratum to: Analysis of overdeepened valleys using the digital elevation model of the bedrock surface of Northern Switzerland}

Peter Jordan

Published online: 4 February 2011

(c) Swiss Geological Society 2011

Erratum to: Swiss J Geosci (2010) 103:375-384

DOI 10.1007/s00015-010-0043-z

During the copy-editing process, the wrong journal was inadvertently inserted in the references by Graf (2003) and Hantke et al. (2003).

The references should read:

Graf, H. R. (2003). Geschichte des unteren Thurtals. Mitteilungen der Thurgauischen Naturforschenden Gesellschaft, 59, 7-29.

Hantke, R., Müller, E., Scheidegger, A. E., \& Wiesmann, A. (2003). Der Molasse-Schuttfächer des Ottenberg und der Lauf der Thur seit dem jüngeren Tertiär. Mitteilungen der Thurgauischen Naturforschenden Gesellschaft, 59, 85-111.

The online version of the original article can be found under doi:10.1007/s00015-010-0043-z.

P. Jordan ( $($ )

Böhringer AG, Mühlegasse 10, 4104 Oberwil, Switzerland

e-mail: peter.jordan@boe-ag.ch 